\title{
Togitare
}

ARTIGO ORIGINAL

\section{SITUAÇÃO CLÍNICO-EPIDEMIOLÓGICA DA SÍFILIS GESTACIONAL EM ANÁPOLIS-GO: UMA ANÁLISE RETROSPECTIVA}

\author{
Ricardo Caldeira dos Santos Filho' ${ }^{1}$ \\ Isabela Cristina Moreira ${ }^{1}$ (1) \\ Lohane Damas Moreira ${ }^{1}$ (1) \\ Lorena Gomes Abadia ${ }^{1}$ \\ Matheus Vallim Machado \\ Mirlene Garcia Nascimento ${ }^{2}$ \\ Constanza Thaise Xavier Silva ${ }^{1}$ (1)
}

\section{RESUMO}

Objetivo: descrever a situação clínica e epidemiológica da sífilis gestacional em Anápolis, Goiás, Brasil, entre os anos de 2012 e 2018.

Método: estudo epidemiológico, descritivo e transversal de natureza quantitativa. Como instrumento de coleta, foram utilizados dados da ficha de notificacão da sífilis gestacional cadastradas no Departamento de Vigilância Epidemiológica. Para análise estatística, foi utilizado o teste quiquadrado com nível de significância $5 \%(p<0,05)$.

Resultados: evidenciou-se aumento dos casos de sífilis gestacional. A assistência foi oferecida a 522 pacientes, entre 19 e 29 anos (58,1\%), com etnia parda $(69,1 \%)$ e com ensino médio incompleto (24,5\%); predominou a sífilis primária (30,8\%) e alto índice de não tratamento do parceiro (57,1\%). Conclusão: dentro do período estudado, houve aumento dos casos de sífilis gestacional e não tratamento do parceiro. Este estudo poderá contribuir para o planejamento de ações de prevenção primária e secundária, visando diminuir a incidência da doença e suas consequências.

DESCRITORES: Sífilis; Gestantes; Epidemiologia; Cuidado Pré-Natal; Testes Sorológicos.

\section{SITUACIÓN CLÍNICA Y EPIDEMIOLÓGICA DE LA SÍFILIS GESTACIONAL EN ANÁPOLIS-GO: UN ANÁLISIS RETROSPECTIVO}

\section{RESUMEN:}

Objetivo: describir la situación clínica y epidemiológica de la sífilis gestacional en Anápolis, Goiás, Brasil, entre los años 2012 y 2018. Método: estudio descriptivo y transversal de carácter cuantitativo. Se utilizaron como instrumento de recogida los datos de la ficha de notificación de sífilis gestacional registrada en el Departamento de Vigilancia Epidemiológica. Para el análisis estadístico se utilizó la prueba de chi-cuadrado con un nivel de significación del $5 \%$ $(p<0,05)$. Resultados: se evidenció un aumento de los casos de sífilis gestacional. La asistencia se ofreció a 522 mujeres, con edades comprendidas entre los 19 y los 29 años (58,1\%), de etnia parda $(69,1 \%$ ) y con estudios secundarios incompletos (24,5\%); predominó la sífilis latente $(58,1 \%)$, seguida de la sífilis primaria $(30,8 \%)$ y un alto índice de no tratamiento de la pareja $(57,1 \%)$. Conclusión: dentro del período estudiado, hubo un aumento de los casos de sífilis gestacional y no tratamiento de la pareja. Este estudio puede contribuir a la planificación de acciones de prevención primaria y secundaria, con el objetivo de disminuir la incidencia de la enfermedad y sus consecuencias. DESCRIPTORES: Sífilis; Mujeres Embarazadas; Epidemiología; Atención Prenatal; Pruebas Serológicas. 
A sífilis é uma infecção sexualmente transmissível (IST) causada pela bactéria espiroqueta Treponema pallidum, de caráter sistêmico e passível de prevenção. Quando não tratada precocemente, pode evoluir para um quadro crônico com sequelas irreversíveis, descrito pela primeira vez em 1905, por Schaudinn e Hoffmann. É transmitida por via sexual e vertical, e raramente via transfusão sanguínea, sendo uma doença de notificação obrigatória ${ }^{(1-2)}$. A transmissão vertical da sífilis pode ocorrer em qualquer período gestacional ou durante o parto, recebendo a denominação de sífilis congênita ${ }^{(3)}$.

Existem várias patologias que podem ser transmitidas durante o período gestacional, porém a sífilis possui as maiores taxas de contágio, variando entre $70 \%$ e $100 \%$ nas fases primária e secundária, e reduzindo para $30 \%$ nas fases latente tardia e terciária da infecção materna ${ }^{(4)}$.

A baixa renda da população com sífilis gestacional é um fator de risco para o aparecimento de sífilis congênita, associada a fatores como baixo peso ao nascer, mortalidade infantil e aumento do número de partos ${ }^{(5)}$. A média de idade entre as gestantes que adquirem sífilis é de 20 a 24 anos, além de estar associada à baixa escolaridade ${ }^{(6)}$.

Os testes utilizados para o diagnóstico da sífilis são divididos em duas categorias: exames diretos e testes imunológicos. Dentre os exames diretos, o principal é a pesquisa direta da bactéria em microscopia de campo escuro, capaz de diagnosticar a doença nos estágios primário e secundário. Já os testes imunológicos - na prática, os mais utilizados dividem-se em treponêmicos (TT) e testes não treponêmicos (TNT). Os testes treponêmicos detectam anticorpos específicos produzidos contra os antígenos da bactéria e são os primeiros a se tornarem reagentes, sendo importantes para a confirmação do diagnóstico, e os testes não treponêmicos detectam anticorpos não específicos anticardiolipina, e podem ser qualitativos ou quantitativos ${ }^{(7)}$.

A definição de testes positivos, treponêmicos ou não, no soro dos recém-nascidos, é limitada em razão da transferência passiva de anticorpos do tipo Imunoglobulina G (IgG) maternos que, no entanto, tendem progressivamente a diminuir até a sua negativação, em alguns meses. No caso de sífilis congênita, ao contrário, os títulos se mantêm ou ascendem, caracterizando uma infecção ativa. O ideal é a realização do teste confirmatório treponêmico na gestante a partir de todo teste não-treponêmico reagente (a partir de títulos de 1:1, o teste não-treponêmico é considerado reagente). $O$ tratamento eficaz contra a bactéria é a penicilina, que é a droga de escolha durante a gravidez ${ }^{(8)}$.

Mundialmente, estima-se que ocorram mais de 11 milhões de novos casos de sífilis por ano, com altos percentuais de incidência em países da América Latina, África e Ásia( ${ }^{(9)}$. No Brasil, onde a doença persiste como um grave problema de saúde pública, houve um aumento no número de notificações e nas taxas epidemiológicas da sífilis gestacional e congênita entre os anos de 2007 e 2016. Em relação à sífilis gestacional, a taxa de detecção foi 2,5 casos $/ 1.000$ nascidos vivos, em 2007 , para 12,4 casos $/ 1.000$ nascidos vivos em $2016^{(10)}$.

A sífilis gestacional é tratável e, por conseguinte, a sífilis congênita pode ser evitada. A ocorrência da sífilis gestacional é indicadora de falhas no pré-natal, no diagnóstico ou tratamento. Na ausência de tratamento, a transmissão vertical da sífilis é elevada, podendo alcançar valores próximos a 100\% nas formas recentes da doença. Contudo, o diagnóstico e tratamento oportuno são altamente eficazes e reduzem a transmissão vertical em até $97 \%$ dos $\operatorname{casos}^{(11)}$.

Observa-se que a sífilis tornou-se um grande problema de saúde pública, sendo de extrema valia que os municípios conheçam a realidade da doença dentro da população para que ações de prevenção e controle sejam adotadas. Portanto, o presente estudo teve 
por objetivo descrever a situação clínico-epidemiológica da sífilis gestacional em Anápolis, Goiás, Brasil entre os anos de 2012 e 2018.

\section{MÉTODO}

Trata-se de um estudo epidemiológico, descritivo e transversal, de natureza quantitativa. De 06 de maio de 2019 a 30 de setembro de 2019, como instrumento de coleta, foram solicitadas as fichas de notificação da sífilis gestacional do Sistema de Informação de Agravos de Notificação (SINAN), vinculados no Departamento de Epidemiologia da Secretaria Municipal de Saúde de Anápolis, relativas ao período de janeiro de 2012 a dezembro de 2018. Esse período foi escolhido devido ao grande número de suspeita de casos levantados pelos sistemas de saúde, com objetivo de realizar uma análise mais detalhada do caso.

As características avaliadas foram: dados sociodemográficos em relação à gestante/ mãe: faixa etária, etnia e escolaridade, e características clínico-epidemiológicas em relação à gestante/mãe: quando ocorreu o diagnóstico de sífilis materna, classificação clínica, realização ou não de TNT e TT juntamente com seus resultados, se o(s) parceiro(s) foi(foram) tratado(s) concomitantemente com a gestante e qual o esquema de tratamento realizado pela gestante e parceiro.

Os critérios de inclusão foram casos de sífilis gestacional notificados no SINAN em Anápolis-GO, Brasil, nos anos de 2012 a 2018, cujas fichas forneceram informações suficientes para análise dos casos e seguimento do estudo. Os critérios de exclusão foram os casos não residentes no município, duplicidades de notificação e fichas ilegíveis.

O programa Excel 2007 foi utilizado para tabulação dos dados e a análise estatística foi realizada pelo programa Statistical Package for Social Sciences (SPSS®) versão 16.0. Para a realização da análise estatística descritiva, foi adotado o teste qui-quadrado. Foi utilizado como nível de significância o valor $5 \%(p<0,05)$ para todas as análises.

A pesquisa seguiu os critérios de éțica e pesquisa com seres humanos, tendo parecer $n^{\circ}$ 2.572.947 aprovado pelo Comitê de Ética em Pesquisa.

\section{RESULTADOS}

Entre 2012 e 2018, foram notificados 522 casos de sífilis em gestantes, com evidente crescimento no número de notificações: passando de 34 casos (6,5\%) no ano de 2012 para 132 casos $(25,3 \%)$ em 2018 (Figura 1$)$. 


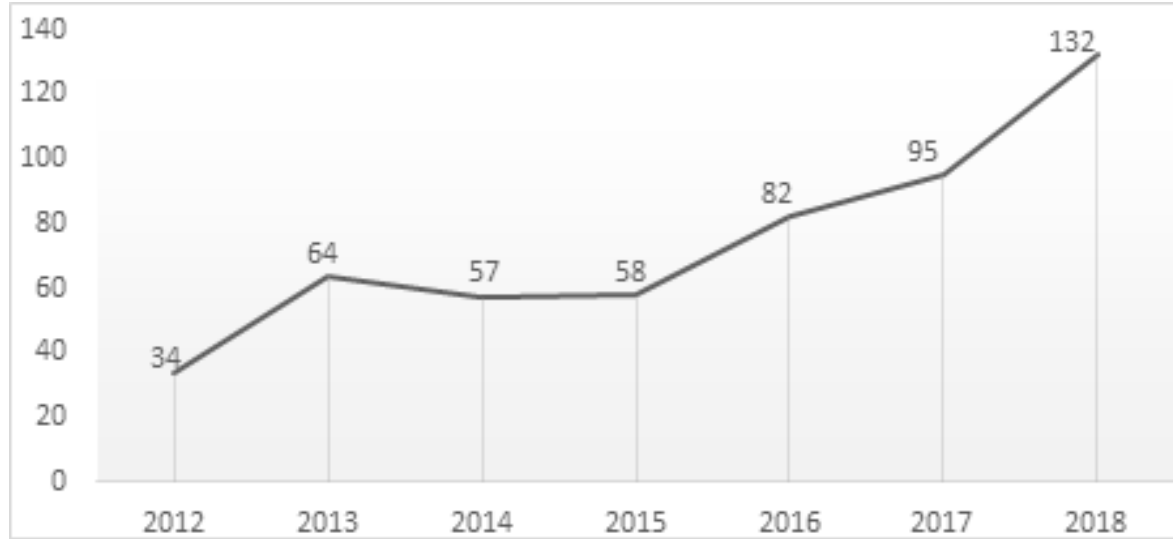

Figura 1 - Distribuição do número de casos notificados de sífilis gestacional entre 2012 e 2018. Anápolis, GO, Brasil, 2020

Fonte: Autores (2020)

De acordo com o perfil sociodemográfico das gestantes, foi verificada uma maior ocorrência na faixa etária de 19 a 29 anos, com 303 (58,1\%) notificações, 361 (69,1\%) são mulheres pardas, 128 (24,5\%) com ensino médio incompleto, como evidenciado na Tabela 1.

Tabela 1 - Distribuição dos casos notificados de sífilis gestacional de 2012 a 2018 por faixa etária, etnia e escolaridade. Anápolis, GO, Brasil, 2020 (continua)

\begin{tabular}{|c|c|c|c|}
\hline Variáveis & $\mathbf{n}$ & $\%$ & $\mathbf{p}$ \\
\hline \multicolumn{4}{|l|}{ Faixa etária } \\
\hline 12 a 18 anos & 95 & 18,2 & 0,607 \\
\hline 19 a 29 anos & 303 & 58,1 & \\
\hline 30 a 39 anos & 103 & 19,7 & \\
\hline 40 a 49 anos & 21 & 4 & \\
\hline \multicolumn{4}{|l|}{ Etnia } \\
\hline Branca & 107 & 20,5 & 0,059 \\
\hline Preta & 36 & 6,9 & \\
\hline Amarela & 8 & 1,5 & \\
\hline Parda & 361 & 69,1 & \\
\hline Indígena & 2 & 0,4 & \\
\hline Ignorada & 6 & 1,2 & \\
\hline Sem dados & 2 & 0,4 & \\
\hline \multicolumn{4}{|l|}{ Escolaridade } \\
\hline Analfabetas & 0 & 0 & 0,001 \\
\hline $1^{\mathrm{a}}$ a $4^{\mathrm{a}}$ série incompleta do ensino fundamental & 27 & 5,2 & \\
\hline $4^{a}$ série completa do ensino fundamental & 19 & 3,6 & \\
\hline $5^{\mathrm{a}}$ a $8^{\mathrm{a}}$ série incompleta do ensino fundamental & 68 & 13 & \\
\hline
\end{tabular}




\begin{tabular}{lcc}
\hline Ensino fundamental completo & 47 & 9 \\
\hline Ensino médio incompleto & 128 & 24,5 \\
\hline Ensino médio completo & 125 & 24 \\
\hline Educação superior incompleto & 9 & 1,7 \\
\hline Educação superior completo & 11 & 2,1 \\
\hline Ignorados & 58 & 11,1 \\
\hline Sem dados & 30 & 5,8 \\
\hline Total & 522 & 100
\end{tabular}

Fonte: Autores (2020)

Foram comparados o ano de notificação dos casos com a idade gestacional das mulheres, evidenciando uma grande mudança no perfil de incidência dos trimestres gestacionais diagnosticados em relação aos anos de estudos ( $p=0,002)$. Em 2012, 2014 e 2015, houve uma maior notificação durante o terceiro trimestre de gestação, já no ano de 2013 foi o segundo trimestre e a partir do ano de 2016, os casos notificados no primeiro trimestre assumiram a primeira posição, mantendo-se assim até 2018 (Figura 2).

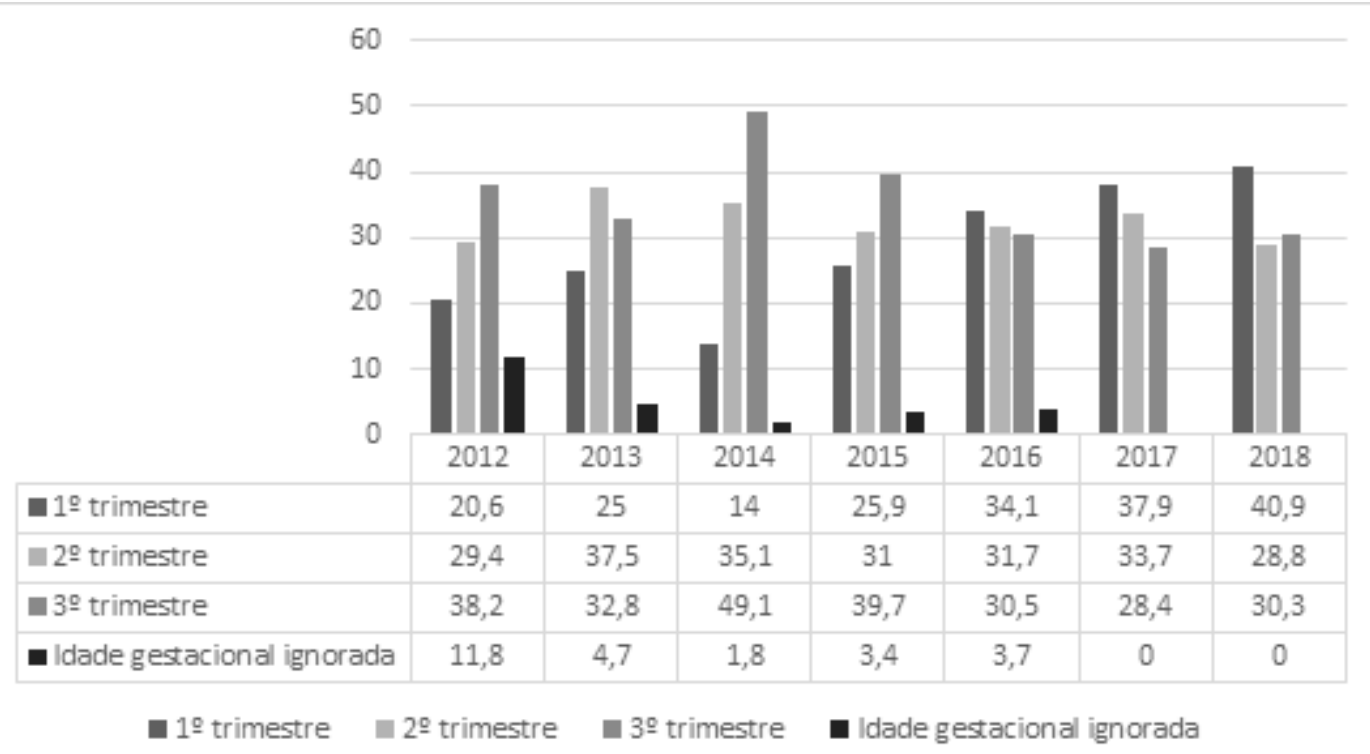

Figura 2 - Análise percentual dos casos de sífilis gestacional por idade gestacional de diagnóstico entre 2012 e 2018. Anápolis, GO, Brasil, 2020

Fonte: Autores (2020)

Observando os casos, de acordo com a classificação clínica, houve predominância da forma latente da sífilis com $303(58,0 \%)$ casos, seguidos da forma primária com 161 (30,8\%). Isto evidencia uma diferença estatisticamente significante entre a classificação clínica e os anos estudados $(p=0,001)$. Em relação aos testes não treponêmicos (TNT) no pré-natal, $443(84,9 \%)$ mulheres realizaram os testes e nove $(1,7 \%)$ tiveram preenchimento da ficha 
ignorado, evidenciando diferença estatisticamente significante $(p=0,001)$ entre os grupos. Já em relação aos testes treponêmicos (TT) realizados no pré-natal, foi demonstrado que $458(87,8 \%)$ mulheres realizaram o teste e seis $(1,1 \%)$ tiveram o preenchimento ignorado da ficha de notificação, evidenciando uma diferença estatisticamente significante $(p=0,005)$ dos casos (Tabela 2).

Tabela 2 - Distribuição dos casos notificados de sífilis gestacional de 2012 a 2018 por critérios clínicos e laboratoriais. Anápolis, GO, Brasil, 2020

\begin{tabular}{|c|c|c|c|}
\hline Variáveis & $\mathrm{n}$ & $\%$ & $\mathrm{p}$ \\
\hline \multicolumn{4}{|l|}{ Classificação clínica } \\
\hline Sífilis primária & 161 & 30,8 & \multirow[t]{6}{*}{0,001} \\
\hline Sífilis secundária & 22 & 4,2 & \\
\hline Sífilis terciária & 11 & 2,1 & \\
\hline Sifilis latente & 303 & 58,1 & \\
\hline Ignorado & 12 & 2,3 & \\
\hline Sem dados & 13 & 2,5 & \\
\hline \multicolumn{4}{|c|}{ Teste não treponêmico no pré-natal } \\
\hline Reagente & 344 & 65,9 & \multirow[t]{4}{*}{0,001} \\
\hline Não reagente & 99 & 19 & \\
\hline Não realizado & 70 & 13,4 & \\
\hline Ignorado & 9 & 1,7 & \\
\hline \multicolumn{4}{|c|}{ Teste treponêmico no pré-natal } \\
\hline Reagente & 433 & 83 & \multirow[t]{4}{*}{0,005} \\
\hline Não reagente & 25 & 4,8 & \\
\hline Não realizado & 58 & 11,1 & \\
\hline Ignorado & 6 & 1,1 & \\
\hline Total & 522 & 100 & \\
\hline
\end{tabular}

Fonte: Autores (2020)

A partir da observação dos diferentes esquemas de tratamento, houve predominância do uso de Penicilina G Benzatina, em 422 (80,9\%) fichas notificadas. Em 68 (13\%) mulheres, o tratamento não foi realizado, enquanto em $15(2,9 \%)$ não houve descrição do esquema empregado, evidenciando uma diferença estatisticamente significante $(p=0,001)$ dos casos em relação ao tratamento escolhido e os anos estudados. No que se refere ao tratamento do parceiro, $298(57,1 \%)$ não foram tratados, $57(10,9 \%)$ fichas foram preenchidas como ignorado e em nove $(1,7 \%)$ fichas não houve preenchimento, evidenciando uma diferença estatisticamente significante $(p=0,001)$ (Tabela 3$)$. 
Tabela 3 - Esquema de tratamento prescrito para a gestante com sífilis e tratamento ou não do parceiro. Anápolis, GO, Brasil, 2020

n $\quad$ p

\begin{tabular}{lccc}
\hline Esquema de tratamento prescrito para a gestante & & & $\mathbf{P}$ \\
\hline Penicilina G benzatina 2.400.000 UI & 154 & 29,5 & \\
\hline Penicilina G benzatina 4.800.000 UI & 40 & 7,7 & \\
\hline Penicilina G benzatina 7.200.000 UI & 228 & 43,7 & 0,001 \\
\hline Outro esquema & 17 & 3,2 & \\
\hline Não realizado & 68 & 13 & \\
\hline Ignorado & 15 & 2,9 & \\
\hline Parceiro tratado & & & \\
\hline Sim & 158 & 30,3 & \\
\hline Não & 298 & 57,1 & \\
\hline Ignorado & 57 & 10,9 & 0,001 \\
\hline Sem dados & 9 & 1,7 & \\
\hline Total & 522 & 100 &
\end{tabular}

Fonte: Autores (2020)

\section{DISCUSSÃO}

Observou-se aumento no número de casos de sífilis gestacional na cidade de Anápolis- GO no período analisado. Atribui-se essa alteração, dentre outros fatores, ao aumento da cobertura de testagem, ampliação do uso de testes rápidos, diminuição do uso de métodos contraceptivos, desabastecimento mundial de penicilina e resistência à administração de penicilina pelos profissionais de saúde na atenção básica, como indicam os dados do Ministério da Saúde ${ }^{(9)}$. Esse aumento da oferta de serviços de assistência pré-natal altera os desfechos das gestações e a sua deficiência pode elevar a mortalidade perinatal em até cinco vezes. Em países em desenvolvimento, pode diminuir a mortalidade materna em até $26 \%(6)$.

O aumento significativo dos casos durante os anos de 2017 e 2018 pode se relacionar, em parte, à mudança da definição de casos, que, em outubro de 2017, passou a considerar notificações durante o pré-natal, parto e puerpério para sífilis gestacional, como forma de diminuir a subnotificação dos casos em gestantes. Ainda assim, a situação no Brasil não difere de outros países em desenvolvimento, apresentando números preocupantes e necessidade de controle das IST ${ }^{(11)}$.

Em relação à faixa etária, a predominância encontrada entre mulheres de 19 a 29 anos ressalta a importância e a necessidade de ampliação de triagem e buscas específicas, além de notificação, para essa fase de maior fertilidade(12). Além disso, a parcela significativa de incidência em mulheres entre 12 e 18 anos por um lado mostra o início cada vez mais precoce da atividade sexual e, por outro, devido à abrangência diagnóstica, mostra uma atenção especial por parte da saúde pública para a população jovem, que se apresenta bastante vulnerável aos riscos relacionados ao comportamento sexual.

Em um estudo acerca do conhecimento sobre IST realizado em Goiânia-GO, ficou 
evidenciada, ainda, falta de conhecimento dos adolescentes sobre determinados pontos das IST e conhecimento básico da grande parte dos avaliados quanto aos métodos de proteção contra essas infecções, demonstrando a importância de campanhas de sensibilização e necessidade de aprimoramento em âmbito escolar, o que pode corroborar para diminuição das taxas de infecção por sífilis e outras IST $^{(13)}$.

Em relação à etnia, a maior incidência de sífilis gestacional em mulheres pardas pode ser atribuída, em parte, às disparidades socioeconômicas enfrentadas por elas: observam-se piores indicadores de atenção pré-natal nas mulheres de etnia parda e preta quando comparadas às brancas ${ }^{(14)}$. Existe uma maior prevalência de sífilis na gestação associada a menores taxas de testagem em mulheres de menor escolaridade e em pretas e pardas, demonstrando como determinantes sociais os critérios etnia e escolaridade e os identificando como fatores de risco para infecção por sífilis gestacional|(15).

Em relação à classificação clínica, houve predominância da sífilis latente seguida da sífilis primária. O boletim epidemiológico de sífilis em Goiás ${ }^{(16)}$ retrata o diagnóstico da sífilis primária em mulheres sendo de difícil detecção, visto que o cancro duro é assintomático e localiza-se, geralmente, em sítios de difícil ou limitada observação, como parede vaginal, cérvix ou períneo; portanto, como perfil de rastreamento, espera-se maior incidência de fase latente em detrimento da fase primária. De acordo ainda com esse boletim, em Goiânia, o perfil com sífilis primária ocupou a primeira posição, seguido de dados ignorado/branco e a latente ocupando a terceira posição, evidenciando possíveis erros de classificação clínica da doença, o que pode levar a condutas terapêuticas equivocadas. O presente estudo encontra-se divergente dos dados encontrados no boletim do estado; além disso, houve uma baixa porcentagem de fichas com preenchimento ignorado/branco.

No que diz respeito ao diagnóstico da sífilis, os testes devem ser feitos em quatro momentos: na primeira consulta do pré-natal (que deve ser realizada prioritariamente no primeiro trimestre); no início do terceiro trimestre (28 semanas); no parto e em caso de abortamento. Nesse sentido, o pré-natal está relacionado com o planejamento estratégico, constituindo um instrumento eficaz na produção de melhoria nas condições de saúde da mulher e sua família ${ }^{(17-18)}$.

Em Goiás, houve melhoria no diagnóstico precoce, mas manutenção do predomínio de diagnóstico tardio da doença, no segundo ou terceiro trimestre de gestação, estando isso possivelmente associado ao início tardio do pré-natal e qualidade da assistência à gestante. Já o município de Anápolis-GO apresentou um perfil semelhante ao goiano até 2015 , com maior taxa de notificações durante o terceiro trimestre. A partir de 2016, houve uma inversão, com maior quantidade de diagnósticos realizados no primeiro trimestre, com essa porcentagem aumentando e chegando a quase $41 \%$ em 2018 , podendo estar relacionado à melhoria da assistência pré-natal e maiores quantidades de rastreios precoces nas gestantes. Contudo, ainda é grande a quantidade de diagnóstico em fases tardias (primária e secundária), mostrando que, apesar de melhoria, ainda há muito a ser feito(19).

Quanto aos exames diagnósticos, uma parcela significativa de gestantes não realizou - TNT. Pelo fato da sífilis gestacional ser silenciosa, com pouco ou nenhum sintoma, a gestante acredita estar totalmente saudável, por isso é importante a realização dos testes diagnósticos que, além do diagnóstico, possibilitam também o tratamento da gestante e seu concepto ${ }^{(20)}$. Este fato pode estar associado ao início da assistência pré-natal tardia e demora no retorno de resultados, levando significativa porção de gestantes a não receberem o resultado do VDRL (do inglês, Venereal Disease Research Laboratory) ainda durante o pré-natal(21). Observa-se, ainda, $70(13,4 \%)$ fichas nas quais não houve realização de TNT mas realização de TT, demonstrando um não seguimento do fluxo diagnóstico estabelecido pelo Ministério da Saúde ${ }^{(22)}$, que é de realização de um TNT como triagem e confirmação diagnóstica ou exclusão de falsos positivos pelo TT, devendo este ser o segundo exame.

Em relação ao tratamento das gestantes, a maioria realizou ao menos uma dose de penicilina G benzatina, o fármaco preconizado pelo Ministério da Saúde ${ }^{(7)}$. Um número 
significativo de gestantes não realizou tratamento, podendo isso repercutir em aumento do número de casos de sífilis congênita e desfechos desfavoráveis. Um fator que pode ter relação com o número de gestantes não tratadas é o desabastecimento da penicilina, desde 2014, que vários países vinham enfrentando, dentre eles o Brasil, devido à falta de matéria-prima para a produção do medicamento, sendo necessárias ações por parte do governo para ajuste do preço do fármaco ${ }^{(23)}$. Segundo o Ministério da Saúde ${ }^{(7)}$, para o tratamento ser considerado eficaz, deve cumprir os seguintes critérios: uso da medicação, esquema indicado para a fase da doença, tratamento instituído até 30 dias antes do parto e tratamento do parceiro.

Quando analisada a forma de tratamento prescrito, observa-se que houve muitas fichas com tratamento inadequado para a fase clínica da doença, apresentando casos com menos e mais doses que o necessário. Em relação à sífilis latente, que apresenta a maior incidência, não é possível fazer uma análise, visto que não há especificação se é latente precoce ou tardia e cada um destes subtipos apresenta um tratamento específico e direcionado ${ }^{(7)}$.

Sobre o parceiro, mais da metade das fichas mostraram que não houve prescrição de tratamento e um número ainda maior de fichas estabelece que não houve o tratamento em si, revelando que, mesmo com a prescrição de tratamento, não houve a terapêutica instituída de forma eficaz. Algumas das causas relatadas nas fichas de notificação para não tratamento do parceiro foram a dificuldade de contato/perda de contato por parte da gestante e recusa de tratamento por parte do parceiro. Percebe-se uma má qualificação do pré-natal, observada pela não realização da sorologia de triagem e do tratamento não realizado ou realizado de forma inadequada do parceiro ${ }^{(24)}$.

Este estudo possui algumas limitações, como o uso de dados secundários, que estão condicionados à qualidade dos registros, além de não permitir estimar o quanto a frequência de subnotificações pode distorcer os resultados encontrados. Entretanto, as bases de dados utilizadas, mesmo com suas limitações, são consideradas confiáveis e de boa qualidade, com produção de informação fidedigna.

\section{CONCLUSÃO}

Este estudo evidenciou aumento da incidência de sífilis gestacional no município de Anápolis-GO no período analisado. Atribui-se essa alteração, dentre outros fatores, ao aumento da cobertura de testagem, ampliação do uso de testes rápidos, diminuição do uso de preservativos e desabastecimento mundial de penicilina. Além disso, percebese a necessidade da difusão de informações sobre prevenção, modo de transmissão e consequências das IST, visto que estas podem trazer desfechos desfavoráveis tanto para a mãe quanto para o concepto.

Nesse sentido, é fundamental que profissionais de saúde e gestores se façam presentes no desenvolvimento de estratégias na melhoria do pré-natal e políticas públicas que abranjam não apenas as gestantes, mas também seus parceiros, uma vez que o não tratamento destes é um obstáculo na interrupção da cadeia de transmissão da sífilis.

Vale ressaltar a necessidade da qualificação adequada dos profissionais de saúde através da educação continuada para o preenchimento correto de prontuários, cartões das gestantes e fichas de notificação, para que o acompanhamento seja feito de modo contínuo e regular.

Percebe-se então a necessidade de novos estudos, bem como sua divulgação para que haja conhecimento pela população e pelo profissionais de saúde sobre a importância das notificações. Ainda são necessárias pesquisas para assegurar, acompanhar e indicar 
possibilidades de atuação do sistema de saúde sobre os casos de sífilis gestacional quanto à prevenção, sintomatologia e tratamento.

\section{REFERÊNCIAS}

1. Arruda LR, Ramos AR dos S. Importance of laboratory diagnosis for congenital syphilis for prenatal. J Manag Prim Health Care [Internet]. 2020 [acesso em 28 jan 2021]; 12:1-18. Disponível em: https://doi. org/10.14295/jmphc.v12.511.

2. Andrade ALMB, Magalhães PVVS, Moraes MM, Tresoldi AT, Pereira RM. Late diagnosis of congenital syphilis: a recurring reality in women and children health care in Brazil. Rev Paul Pediatr. [Internet]. 2018. [acesso em 14 abr 2019]; 36(3):376-81. Disponível em: https://doi.org/10.1590/19840462/;2018;36;3;00011.

3. Moreira KFA, Oliveira DM de, Alencar LN de, Cavalcante DFB, Pinheiro A de S, Orfão NH. Perfil dos casos notificados de sífilis congênita. Cogit. Enferm. [Internet]. 2017 [acesso em 21 maio 2019]; 22(2):110. Disponível em: http://dx.doi.org/10.5380/ce.v22i2.48949.

4. Rocha CC, Lima TOS, Silva RAN, Abrão RK. Approaches to congenital syphilis. Res., Soc. Dev [Internet]. 2020 [acesso em 28 jan 2021]; 9(8):1-18. Disponível em: https://doi.org/10.33448/rsd-v9i8.6820.

5. Cabral BTV, Dantas J da C, Silva JA da, Oliveira DA de. Sífilis em gestante e sífilis congênita: um estudo retrospectivo. Rev. Ciênc. Plur.[Internet]. 2017 [acesso em 18 maio 2019]; 3(3):32-44. Disponível em: https://www.periodicos.ufrn.br/rcp/article/view/13145/9351.

6. Conceição HN da, Câmara JT, Pereira BM. Epidemiological and spatial analysis of cases of gestational and congenital syphilis. Saúde Debate [Internet]. 2019 [acesso em 28 jan 2021]; 43(123):1145-58.

Disponível em: http://dx.doi.org/10.1590/0103-1104201912313.

7. Ministério da Saúde (BR) Secretaria de Vigilância em Saúde. Departamento de DST, Aids e Hepatites Virais. Protocolo clínico e diretrizes terapêuticas. Atenção Integral às pessoas com Infecções Sexualmente transmissíveis (IST). [Internet]. Brasília: Ministério da Saúde, 2015 [acesso em 12 set 2019]. Disponível em: http://bvsms.saude.gov.br/bvs/publicacoes/protocolo clinico diretrizes terapeutica atencao integral pessoas infeccoes sexualmente transmissiveis.pdf.

8. Ribeiro MD, Barreto IFB e, Ramos LR, Pereira CG, Silva JL, Chiapeta MG. Retrospective analysis of the clinical development of congenital syphilis cases treated with ceftriaxone or penicilline in the year of 2016 to june 2018 at the reference ambulatory in the Teresopolis city. Revista Jopic. [Internet]. 2020 [acesso em 28 jan 2021]; 3(6):5-14. Disponível em: https://www.unifeso.edu.br/revista/index.php/jopic/article/ view/1905/806.

9. PAHO (Pan American Health Organization). Elimination of Mother-to-Child Transmission of HIV and Syphilis in the Americas - Update 2015. [Internet]. Washington, DC; 2015 [acesso em 14 set 2019]. Disponível em: https://iris.paho.org/bitstream/handle/10665.2/18372/9789275118702 eng. pdf? sequence=3\&isAllowed=y.

10. Brasil. Ministério da Saúde. Secretaria de Vigilância em Saúde. Departamento de DST, Aids e Hepatites Virais. Boletim Epidemiológico - Sífilis. [Internet]. Brasília, DF; 2016 [acesso em 23 abr 2019]. Disponível em: http://www.aids.gov.br/pt-br/pub/2016/boletim-epidemiologico-de-sifilis-2016.

11. Domingues RMSM, Leal $M$ do $C$. Incidência da sífilis congênita e fatores associados à transmissão vertical da sífilis: dados do estudo Nascer no Brasil. Cad. Saúde Pública [Internet]. 2016 [acesso em 19 set 2019]; 32(6):1-12. Disponível em: http://dx.doi.org/10.1590/0102-311X00082415.

12. Marques JVS, Alves BM, Marques MVS, Arcanjo FPN, Parente CC, Vasconcelos RL. Perfil epidemiológico da sífilis gestacional: clínica e evolução de 2012 a 2017. SANARE, Sobral. [Internet]. 2018 
[acesso em 30 abr 2019]: 17(2):13-20. Disponível em: https://doi.org/10.36925/sanare.v17i2.1257.

13. Cunha M dos P, Oliveira BFR de, Oliveira ICM de, Praxedes LKS, Reis AA da S. Análise do conhecimento sobre DSTs/AIDS entre adolescentes em Goiânia, Goiás. UNINCOR. [Internet]. 2016 [acesso em 18 out 2019]; 14(2):650-8. Disponível em: http://dx.doi.org/10.5892/ruvrd.v14i2.2856.

14. Leal M do C, Gama SGN da, Pereira APE, Pacheco VE, Carmo CN do, Santos RV. The color of pain: racial iniquities in prenatal care and childbirth in Brazil. Cad. Saúde Pública. [Internet]. 2017 [acesso em 21 set 2019]; 33(Suppl 1):1-17. Disponível em: https://doi.org/10.1590/0102-311×00078816.

15. Morais TR, Feitosa WG, Oliveira IC, Girão MMF, Sales WS, Brito EAS, et al. Intersectionalities in health: prevalence of gestational syphilis in black women and pardas in Brazil. Id on Line Rev. Mult. Psic. [Internet]. 2019 [acesso em 28 jan 2021]; 13(45):670-9. Disponível em: https://doi.org/10.14295/idonline. v13i45.1772.

16. Secretaria do Estado de Goiás. Superintendência de Políticas de Atenção Integral à Saúde. Gerência de Programas Especiais. Coordenação Estadual de IST/AIDS. Situação epidemiológica da sífilis adquirida, sífilis em gestantes e sífilis congênita no Estado de Goiás. [Internet]. Goiânia, 2017 [acesso em 26 nov 2019]. Disponível em: https://www.saude.go.gov.br/images/imagens migradas/2019/03/boletimepidemiologicosifilis-2018.pdf.

17. Cavalcante PA de M, Pereira RB de L, Castro JGD. Sífilis gestacional e congênita em Palmas, Tocantins, 2007-2014. Epidemiol. Serv. Saude. [Internet]. 2017 [acesso em 14 set 2019]; 26(2):255-64. Disponível em: https://doi.org/10.5123/s1679-49742017000200003.

18. Araújo $E$ da $C$, Monte $P C B$, Haber ANC de A. Avaliação do pré-natal quanto à detecção de sílilis e HIV em gestantes atendidas em uma área rural do estado do Pará, Brasil. Rev Pan-Amaz Saude [Internet]. 2018 [acesso em 15 abr 2019]; 9(1):33-9. Disponível em: http://dx.doi.org/10.5123/s2176$\underline{62232018000100005 .}$

19. Nunes PS, Zara AL de SA, Rocha DFN de C, Marinho TA, Mandacarú PMP, Turchi MD. Sífilis gestacional e congênita e sua relação com a cobertura da Estratégia Saúde da Família, Goiás, 20072014: um estudo ecológico. Epidemiol. Serv. Saude [Internet]. 2018 [acesso em 03 mar 2019]; 27(4):1-10. Disponível em: https://doi.org/10.5123/s1679-49742018000400008.

20. Silva EC da, Tupinambá MR, Silva FASD da, Vieira JR, Borges SCR, Nascimento LS do. Resultados de sorologia para casos de sífilis em campanha de município do norte do Brasil. Rev Pan-Amaz Saude [Internet]. 2016 [acesso em 18 maio 2019]; 7(1):39-43. Disponível em: http://scielo.iec.gov.br/pdf/rpas/ v7n1/v7n1a05.pdf.

21. Cardoso ARP, Araújo MAL, Cavalcante M do S, Frota MA, Melo SP de. Análise dos casos de sífilis gestacional e congênita nos anos de 2008 a 2010 em Fortaleza, Ceará, Brasil. Ciênc. saúde coletiva [Internet]. 2018 [acesso em 07 set 2019]; 23(2):563-74. Disponível em: https://doi.org/10.1590/141381232018232.01772016.

22. Ministério da Saúde (BR) Secretaria de Vigilância em Saúde. Departamento de Vigilância, Prevenção e Controle das Doenças Sexualmente Transmissíveis, Aids e Hepatites Virais. Manual técnico para diagnóstico da sífilis. [Internet]. Brasília: Ministério da Saúde, Secretaria de Vigilância em Saúde, 2016 [acesso em 25 nov 2019]. Disponível em: http://www.aids.gov.br/pt-br/pub/2016/manual-tecnico-paradiagnostico-da-sifilis.

23. Ministério da Saúde (BR). Medida Provisória viabilizará compra de penicilina para combater sífilis [Internet]. Brasília, DF; 2016 [acesso em 16 abr 2020]. Disponível em: http://www.cosemsrn.org.br/ noticias/medida-provisoria-viabilizara-compra-de-penicilina-para-combater-sifilis/.

24. Amaral E. Sífilis na gravidez e óbito fetal: de volta para o futuro. Rev Bras Ginecol Obstet. [Internet]. 2012 [acesso em 14 nov 2019]; 34(2):52-5. Disponível em: https://doi.org/10.1590/S010072032012000200002. 
COMO REFERENCIAR ESTE ARTIGO:

Santos Filho RC dos, Moreira IC, Moreira LD, Abadia LG, Machado MV, Nascimento MG, et al. Situação clínicoepidemiológica da sífilis gestacional em Anápolis-GO: uma análise retrospectiva. Cogit. Enferm. [Internet]. 2021 [acesso em "colocar data de acesso, dia, mês abreviado e ano"]; 26. Disponível em: http://dx.doi.org/10.5380/ ce.v26i0.75035.

Recebido em: 06/07/2020

Aprovado em: 02/02/2021

Editora associada: Tatiane Herreira Trigueiro

Autor Correspondente:

Constanza Thaise Xavier Silva

Centro Universitário de Anápolis - Anápolis, GO, Brasil

E-mail: constanza.silva@unievangelica.edu.br

Contribuição dos autores:

Contribuições substanciais para a concepção ou desenho do estudo; ou a aquisição, análise ou interpretação de dados do estudo - RCSF, ICM, LDM, LGA, MVM, MGN, CTXS

Elaboração e revisão crítica do conteúdo intelectual do estudo - RCSF, ICM, LDM, LGA, MVM, MGN, CTXS

Aprovação da versão final do estudo a ser publicado - RCSF, ICM, LDM, LGA, MVM, MGN, CTXS

Responsável por todos os aspectos do estudo, assegurando as questões de precisão ou integridade de qualquer parte do estudo - RCSF, ICM, LDM, LGA, MVM, MGN, CTXS

ISSN 2176-9133

Copyright $\odot 2021$ Este é um artigo em acesso aberto distribuído nos termos da Licença Creative Commons Atribuição, que permite o uso irrestrito, a distribuição e reprodução em qualquer meio desde que o artigo original seja devidamente citado. 